Europhysics Letters

PREPRINT

\title{
Surfing on a critical line: Rejuvenation without chaos, Memory without a hierarchical phase space
}

\author{
Ludovic Berthier and Peter C. W. Holdsworth \\ Laboratoire de Physique, ENS-Lyon and CNRS, 46 Allée d'Italie, 69007 Lyon, France
}

PACS. 05.70.Ln - Nonequilibrium thermodynamics, irreversible processes.

PACS. 75.40.Gb - Dynamic properties (dynamic susceptibility, spin waves, spin diffusion, dynamic scaling, etc.).

PACS. 64.60.Ht - Dynamic critical phenomena.

\begin{abstract}
The dynamic behaviour of glassy materials displays strong nonequilibrium effects, such as ageing in simple protocols, memory, rejuvenation and Kovacs effects in more elaborated experiments. We show that this phenomenology may be easily understood in the context of the nonequilibrium critical dynamics of non-disordered systems, the main ingredient being the existence of an infinite equilibrium correlation length. As an example, we analytically investigate the behaviour of the 2D XY model submitted to temperature protocols similar to experiments. This shows that typical glassy effects may be obtained by 'surfing on a critical line' without invoking the concept of temperature chaos nor the existence of a hierarchical phase space, as opposed to previous theoretical approaches. The relevance of this phenomenological approach to glassy dynamics is finally discussed.
\end{abstract}

...comme des amateurs de surf qui glissaient sur la vague. Vassili Axionov, Une brûlure.

Experiments on a large class of glassy materials demonstrate intriguing similarities in their nonequilibrium dynamic properties. Structural —in particular polymeric - glasses are the paradigm, and have been studied in a systematic way over a long period time [1]. Interest in ageing experiments grew with the realization that very similar effects are observed in spin glasses [2]. More recently, the family of glassy systems has been much enlarged to include systems as different as dirty type-II superconductors [3], several complex fluids 仼, disordered dielectrics [5, 6] and ferromagnets [7], electron glasses [8], and granular materials [9].

These systems have in common that, in a part of their phase space, they cannot be equilibrated within the experimental time window: they undergo a 'glass' or 'jamming' transition. As a result, they exhibit nonequilibrium dynamics, where the whole history of the sample becomes relevant [10]. The conceptually simplest experiment performed in glassy materials consists of a sudden quench of the system into its glassy phase at the initial time $t_{w}=0$. The resulting ageing behaviour is best observed through the measurement of a two-time quantity, $C\left(t, t_{w}\right)$, typically a correlation function or a linear susceptibility. Experiments show that time translation invariance is absent, i.e. $C\left(t, t_{w}\right) \neq C\left(t-t_{w}\right)$. Remarkably, they also show that the generic scaling behaviour $C\left(t, t_{w}\right) \sim \mathcal{C}\left(t / t_{w}\right)$ is very often observed, implying that the relaxation time $t_{\text {rel }}$ of the system is of the order of its age, $t_{\text {rel }} \sim t_{w}$.

This ageing phenomenology can be explained by models of coarsening of domains of equivalent thermodynamic phases [10,11]. The canonical example is a ferromagnet quenched into

(C) EDP Sciences 
its ordered phase which exhibits ageing, due to the growth of ferromagnetic domains of different orientations. A length scale $\ell_{T}(t)$, over which equilibrium is established, grows with time $t$ at temperature $T$, leading to the above scaling behaviour [11]. However, such simple models fail to explain the spectacular 'memory' and 'rejuvenation' effects [12] observed in more elaborate experimental protocols unless complemented in some way. One approach is to include temperature chaos [13], which amounts to a great sensitivity of equilibrium states to temperature changes, as first postulated in the context of spin glasses 14. The very existence of temperature chaos in this sense is however still debated, with rather negative conclusions.

Ageing may alternatively be viewed as the search by the system of the deepest 'traps' of a complex energy landscape [15]. Extending this approach to a hierarchical structure of the traps allows for a qualitative understanding of temperature cycling experiments [16]. Multitrap models of this kind are studied in Ref. [17]. This approach has however the disadvantage of not providing a real space description of the problem. Ageing is also captured in a more microscopic way by the exact solution of several mean-field disordered models [10,18]. These do account for rejuvenation and memory [18], but the explanation relies on the notion of dynamic ultrametricity, which is incompatible with experimental data [18].

In this paper we show that a phenomenological domain growth approach may be used to interpret $T$-cycling experiments, provided the dynamics is considered at criticality. For a quench into an ordered phase, the equilibrium correlation length $\xi_{\text {eq }}$ is microscopic and therefore decoupled from $\ell_{T}(t)$. However, the key point here is that quenching to a critical point, one has $\xi_{\mathrm{eq}}=\infty$, with the result that equilibrium fluctuations occur on all length scales up to the dynamic correlation length $\xi(t)=\ell_{T}(t)$. Temperature cycling along a line of critical points thus affects critical fluctuations of all length scales between the lattice spacing and the system size. This allows for both rejuvenation and memory effects without making the assumption of temperature chaos, or evolution in a hierarchical phase space. This may also be viewed as a concrete realization of the multi-length scale scenario discussed by Bouchaud [15]. As an example, the 2D XY model is analytically considered, although the physical interpretation of the results is of more general validity. Finally, we argue that this phenomenological approach may be relevant to many glassy materials.

Ageing at a critical point. - The $2 \mathrm{D} \mathrm{XY}$ model presents a line of critical points in the temperature range $T \in\left[0, T_{\mathrm{KT}}\right]$ where $T_{\mathrm{KT}}$ is the temperature of the Kosterlitz-Thouless transition. The model describes a two-component order parameter $\boldsymbol{\varphi}(\boldsymbol{x}, t)$ in a bidimensional space. The properties of the low temperature phase, $0<T<T_{\mathrm{KT}}$, can be described by the spin-wave approximation [19]

$$
H[\theta]=\frac{\rho(T)}{2} \int \mathrm{d}^{2} \boldsymbol{x}|\nabla \theta|^{2},
$$

with $\varphi=e^{i \theta}$ and where $\rho(T)$ is the spin-wave stiffness. The dynamics is modelled by a Langevin equation

$$
\frac{\partial \theta(\boldsymbol{x}, t)}{\partial t}=-\frac{\delta H[\theta]}{\delta \theta(\boldsymbol{x}, t)}+\zeta(\boldsymbol{x}, t)
$$

The last term is the thermal noise, described by a Gaussian variable of zero mean and variance $\left\langle\zeta(\boldsymbol{x}, t) \zeta\left(\boldsymbol{x}^{\prime}, t^{\prime}\right)\right\rangle=4 \pi \eta(T) \rho(T) \delta\left(\boldsymbol{x}-\boldsymbol{x}^{\prime}\right) \delta\left(t-t^{\prime}\right)$, where $\eta(T)$ is the usual critical exponent, linked to the spin-wave stiffness by $2 \pi \eta(T) \rho(T)=T$. The dynamic behaviour is analysed through the two-point correlation function $C(\boldsymbol{r}, t)=\langle\boldsymbol{\varphi}(\boldsymbol{x}, t) \cdot \boldsymbol{\varphi}(\boldsymbol{x}+\boldsymbol{r}, t)\rangle$, and the two-time autocorrelation function $C\left(t, t_{w}\right)=\left\langle\boldsymbol{\varphi}(\boldsymbol{x}, t) \cdot \boldsymbol{\varphi}\left(\boldsymbol{x}, t_{w}\right)\right\rangle$. Due to the Gaussian character of 
the Hamiltonian (11), these quantities follow straightforwardly from the computation of the angle-angle correlation function $C_{\theta}\left(\boldsymbol{k}, t, t_{w}\right)=\left\langle\theta(\boldsymbol{k}, t) \theta\left(-\boldsymbol{k}, t_{w}\right)\right\rangle$.

The ageing dynamics of the model was discussed in details in Refs [20 22]. In particular, we recall that topological defects are not described by the spin wave approximation (11), although they can influence the dynamics following a quench [21]. We thus choose initial conditions such that no vortices are initially present in the system, with $C_{\theta}(\boldsymbol{k}, 0,0)=2 \pi \eta\left(T_{i}\right) / k^{2}$, where $T_{i}$ is the initial temperature [22]. This implies $C(\boldsymbol{r}, 0) \sim(r / a)^{-\eta\left(T_{i}\right)}$ at large distances. The UV cutoff $a$ is introduced through the factor $e^{-k^{2} a^{2}}$ in all integrals over the Fourier space, simulating the lattice spacing. Although the correlation length in the initial state is not defined, the correlation function decays very rapidly if $\eta\left(T_{i}\right)$ is chosen to have a large value.

The dynamics following a quench to temperature $T_{1}$ at time $t=0$ is solved by Fourier transforming (2), using (11). For the angle-angle correlation one obtains

$$
C_{\theta}\left(\boldsymbol{k}, t, t_{w}\right)=\frac{2 \pi \eta_{1}}{k^{2}} \exp \left[-\left(k \ell_{T_{1}}\left(t-t_{w}\right)\right)^{2}\right]+\frac{2 \pi\left(\eta_{i}-\eta_{1}\right)}{k^{2}} \exp \left[-\left(k \ell_{T_{1}}\left(t+t_{w}\right)\right)^{2}\right]
$$

We define the dynamic correlation length $\ell_{T_{k}}(t)=\left(\rho_{k} t\right)^{1 / z}, z=2$, together with the notation $\eta_{k}=\eta\left(T_{k}\right)$ and $\rho_{k}=\rho\left(T_{k}\right)$. Two-point and two-time correlation functions easily follow from (3). For times such that $\ell_{T_{1}}\left(t-t_{w}\right) \gg a$, one finds

$$
\begin{aligned}
C(\boldsymbol{r}, t) & \simeq \exp \left[-\eta_{1} G\left(\frac{r}{2 a}\right)+\left(\eta_{1}-\eta_{i}\right) G\left(\frac{r}{2 \sqrt{2} \ell_{T_{1}}(t)}\right)\right], \\
C\left(t, t_{w}\right) & \simeq\left(\frac{a}{\ell_{T_{1}}\left(t-t_{w}\right)}\right)^{\eta_{1}}\left(\frac{\ell_{T_{1}}^{2}(t)+\ell_{T_{1}}^{2}\left(t_{w}\right)}{4 \ell_{T_{1}}(t) \ell_{T_{1}}\left(t_{w}\right)}\right)^{\left(\eta_{1}-\eta_{i}\right) / 2},
\end{aligned}
$$

where $G(x)=\frac{1}{2}\left(C+2 \ln x+\int_{x^{2}}^{\infty} \mathrm{d} t \frac{e^{-t}}{t}\right) ; C$ is the Euler constant. For distances $a \ll r \ll$ $\ell_{T_{1}}(t)$, equilibrium behaviour is obtained at $T_{1}, C(\boldsymbol{r}, t) \sim(r / a)^{-\eta_{1}}$, while at large distances, $\ell_{T_{1}}(t) \ll r$, one has $C(\boldsymbol{r}, t) \sim(r / a)^{-\eta_{i}}\left(a / \ell_{T_{1}}(t)\right)^{\eta_{1}-\eta_{i}}$, which is the same power law behaviour as in the initial state, with a time-dependent multiplicative factor 22. Similarly, the two-time correlation function displays an initial equilibrium behaviour $C\left(t, t_{w}\right) \sim\left(t-t_{w}\right)^{-\eta_{1} / 2}$, which is interrupted after a time $t-t_{w} \sim t_{w}$, when the relevant scaling variable becomes $\ell_{T_{1}}(t) / \ell_{T_{1}}\left(t_{w}\right)$.

The dynamics is thus interpreted as consisting of two types of critical fluctuations 21, 23]. For wavevectors $k \ell_{T_{1}}(t) \gg 1$, the equilibrium state at $T_{1}$ has been reached, while fluctuations with $k \ell_{T_{1}}(t) \ll 1$ are still very near to their initial nonequilibrium state. The ubiquitous $t / t_{w}$ scaling is thus obtained as a consequence of a growing equilibration length in the system, as in ordinary coarsening [10]. The only difference is that here the correlation length is fixed to $\ell_{T}(t)$, while in the former it is microscopic.

Rejuvenation and memory effects. - In temperature cycling experiments, the system is first quenched to a temperature $T_{1}$ where it ages. After a time $t_{1}$, the temperature is shifted to $T_{2}<T_{1}$. It is observed that the ageing is restarted by the temperature change: this is the rejuvenation effect. Then, at time $t_{2}>t_{1}$, the temperature is changed back to $T_{1}$. After a short transient time the ageing is observed to be the same as if $t_{2}=t_{1}$, that is, as if the temperature cycle had not existed: this is the memory effect (see Fig. 11). The coexistence of these two effects was popularized by the 'dip-experiment' proposed in Ref. [24], which has since been largely used, showing again very similar results in different materials [2, 3, $507,16,25]$.

The rejuvenation effect can be conveniently demonstrated by taking the limit $t_{1} \rightarrow \infty$, in which case the system starts from an equilibrated configuration at $T_{1}$ and is quenched to $T_{2}$. This is in fact precisely the situation considered in the previous section if the changes $T_{i} \rightarrow T_{1}$ 


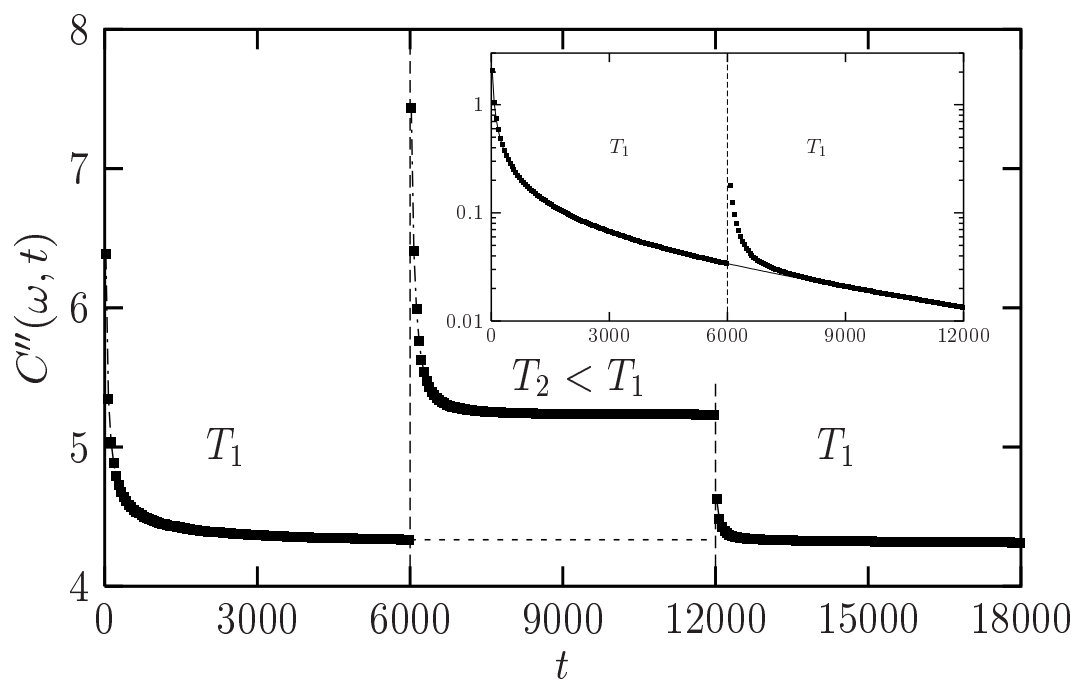

Fig. 1 - Evolution of the imaginary part of the Fourier transform of the autocorrelation function in a temperature cycling. Parameters are: $a=1, \omega=50, \eta_{i}=2.0, t_{1}=6000, \eta_{1}=0.7, \rho_{1}=1.0$, $t_{2}=12000, \eta_{2}=0.35, \rho_{2}=0.05$. Inset: the time interval at $T_{2}$ has been dropped out. To better demonstrate the memory, $C^{\prime \prime}(\omega, t)-C^{\prime \prime}(\omega, \infty)$ has been plotted in a lin-log scale, the full line being the behaviour with $t_{2}=t_{1}$, i.e. without the cycle.

and $T_{1} \rightarrow T_{2}$ are made. Thus, Eqs. (4) illustrate the fact that ageing is restarted when the temperature is changed along the line of critical points. Keeping $t_{1}$ finite, the angle-angle correlation becomes, at times $t>t_{w}>t_{1}$,

$$
\begin{aligned}
C_{\theta}\left(\boldsymbol{k}, t, t_{w}\right) & =\frac{2 \pi \eta_{2}}{k^{2}} \exp \left[-\left(k \ell_{T_{2}}\left(t-t_{w}\right)\right)^{2}\right]+\frac{2 \pi\left(\eta_{1}-\eta_{2}\right)}{k^{2}} \exp \left[-\left(k \ell_{T_{2}}\left(t+t_{w}-2 t_{1}\right)\right)^{2}\right] \\
& +\frac{2 \pi\left(\eta_{i}-\eta_{1}\right)}{k^{2}} \exp \left[-\left(k \ell_{T_{2}}\left(t+t_{w}-2 t_{1}\right)\right)^{2}\right] \exp \left[-2\left(k \ell_{T_{1}}\left(t_{1}\right)\right)^{2}\right]
\end{aligned}
$$

When $t_{1} \rightarrow \infty$, the last term vanishes and the results of the previous section are indeed recovered. On the contrary, when $\ell_{T_{2}}\left(t-t_{1}\right) \gg \ell_{T_{1}}\left(t_{1}\right)$, the last two terms combine to give an ageing behaviour as if the system was directly quenched to $T_{2}$. Equation (5) shows that fluctuations with $k \ell_{T_{1}}\left(t_{1}\right) \gg 1$ age at $T_{2}$ as if they were quenched from $T_{1}$ (the last exponential makes the last term vanish), whereas those with $k \ell_{T_{1}}\left(t_{1}\right) \ll 1$ age at $T_{2}$ as in a quench from $T_{i}$. In this case the last two terms may indeed be combined when the last exponential is $\sim 1$ to make the contribution proportional to $\eta_{1}$ disappear.

Physically, rejuvenation results from the fact that when the temperature is shifted to $T_{2}<T_{1}$, all fluctuations fall out of equilibrium. Fluctuations such that $k \ell_{T_{1}}\left(t_{1}\right) \gg 1$ have to adapt their Boltzmann weight to the new temperature, while for $k \ell_{T_{1}}\left(t_{1}\right) \ll 1$, no equilibrium had been found at the previous temperature. Thus even when $t_{1}=\infty$, ageing is restarted by a temperature change. We emphasize that the effect is absent in a standard coarsening below $T_{c}$, where the reequilibration of thermal fluctuations following the shift to $T_{2}$ is very fast, with an associated time scale $t_{\mathrm{r}}$ given by $\ell_{T_{2}}\left(t_{\mathrm{r}}\right) \sim \xi_{\text {eq }}\left(T_{1}\right) \ll \ell_{T_{1}}\left(t_{1}\right)$.

The memory effect can be seen as the temperature is changed back to $T_{1}$ at total time $t_{2}$, 
with $\ell_{T_{2}}\left(t_{2}-t_{1}\right) \ll \ell_{T_{1}}\left(t_{1}\right)$. The angle-angle correlation function reads now

$$
\begin{aligned}
C_{\theta}\left(\boldsymbol{k}, t, t_{w}\right) & =\frac{2 \pi \eta_{1}}{k^{2}} e^{-\left(k \ell_{T_{1}}\left(t-t_{w}\right)\right)^{2}}+\frac{2 \pi\left(\eta_{2}-\eta_{1}\right)}{k^{2}} e^{-\left(k \ell_{T_{1}}\left(t+t_{w}-2 t_{2}\right)\right)^{2}}\left[1-e^{-2\left(k \ell_{T_{2}}\left(t_{2}-t_{1}\right)\right)^{2}}\right] \\
& +\frac{2 \pi\left(\eta_{i}-\eta_{1}\right)}{k^{2}} e^{-2\left(k \ell_{T_{1}}\left(t_{1}\right)\right)^{2}} e^{-2\left(k \ell_{T_{2}}\left(t_{2}-t_{1}\right)\right)^{2}} e^{-\left(k \ell_{T_{1}}\left(t+t_{w}-2 t_{2}\right)\right)^{2}} .
\end{aligned}
$$

To understand this result, three types of fluctuations have to be considered.

(i) $k^{-1} \ll \ell_{T_{2}}\left(t_{2}-t_{1}\right)$. These fluctuations are equilibrated at $T_{2}$ and have to reequilibrate at $T_{1}$. This can be seen by setting $e^{-2\left(k \ell_{T_{2}}\left(t_{2}-t_{1}\right)\right)^{2}} \sim 0$ in Eq. (6), from which one finds an equation similar to (3), describing a shift from $T_{2}$ to $T_{1}$. The reequilibration takes place on a time scale $t_{\mathrm{m}}$ such that $\ell_{T_{1}}\left(t_{\mathrm{m}}\right) \sim \ell_{T_{2}}\left(t_{2}-t_{1}\right) \ll \ell_{T_{1}}\left(t_{1}\right)$, i.e. $t_{\mathrm{m}} \ll t_{1}$. This is the short initial transient observed in experiments and in Fig. 1.

(ii) $\ell_{T_{2}}\left(t_{2}-t_{1}\right) \ll k^{-1} \ll \ell_{T_{1}}\left(t_{1}\right)$. Only the first term of Eq. (6) survives. These fluctuations had no time to equilibrate at $T_{2}$ but were equilibrated at $T_{1}$ before the cycle. Hence, they undergo equilibrium dynamics at $T_{1}$ immediatly after the cycle. It is in these length scales that the memory is conserved.

(iii) $\ell_{T_{1}}\left(t_{1}\right) \ll k^{-1}$. Only the last term in Eq. (6) survives for these wavevectors. Taking $e^{-2\left(k \ell_{T_{1}}\left(t_{1}\right)\right)^{2}} \sim 1$ and $e^{-2\left(k \ell_{T_{2}}\left(t_{2}-t_{1}\right)\right)^{2}} \sim 1$, Eq. (3) is recovered, describing a quench from $T_{i}$ to $T_{1}$. These length scales retained their initial nonequilibrium state throughout the temperature cycle and will thus equilibrate at $T_{1}$ through the further growth of $\ell_{T_{1}}$.

As a consequence, after the transient time $t_{\mathrm{m}}$, the dynamics appears as if the cycle at $T_{2}$ was absent, accounting for the memory effect observed in experiments. We show in Fig. 1 the behaviour of the imaginary part of the Fourier transform of the autocorrelation function $C(\omega, t)=\int_{0}^{t} \mathrm{~d} t^{\prime} C\left(t, t^{\prime}\right) e^{i \omega\left(t-t^{\prime}\right)}$ in a temperature cycle. At equilibrium, this function is directly related by the fluctuation-dissipation theorem to the linear susceptibility $\chi(\omega, t)$, which is the quantity measured experimentally. The figure demonstrates that the rejuvenation and memory effects are quantitatively accounted for by the 2D XY model. The two-time autocorrelation function is computed from Eqs. (5) and (6), as was done to obtain Eq. (4) from Eq. (3). As it is rather complicated, we do not report it here, its interpretation being the same as that developed above.

Interestingly, the same effects could be observed in a positive $T$-cycle, $T_{2}>T_{1}$, provided $\ell_{T_{2}}\left(t_{2}-t_{1}\right) \ll \ell_{T_{1}}\left(t_{1}\right)$. This feature is in qualitative contradiction with the hierarchical phase space picture [16], and experiments could thus distinguish between the two approaches.

Kovacs effect. - In the literature of structural glasses, 'memory effect' has a different meaning [1, 26]. Here, the system is first quenched to a given temperature $T_{1}$. The slow nonequilibrium evolution of a one-time quantity $V(t)$ (volume, refraction index) is followed. The temperature is then raised at time $t_{1}$ to a value $T_{2}$ such that, at equilibrium, $V$ would have the value it has just before the shift, i.e. $V\left(t_{1}\right)=V_{\text {eq }}\left(T_{2}\right)$. Whereas it would be naively expected that $V\left(t>t_{1}\right)=$ const, a nontrivial non-monotonic behaviour is observed instead. To avoid confusion, we will refer to this effect as the Kovacs effect [26]. Again, we note that it was observed in several materials [1,5,9,26, 29.

Within the 2D XY model, one follows the behaviour of the energy density $e(t)$ in a protocol $T_{i} \rightarrow T_{1}$ at $t=0$ and $T_{1} \rightarrow T_{2}$ at $t_{1}$, with $T_{i}>T_{2}>T_{1}$. For times such that $\ell_{T_{2}}\left(t-t_{1}\right) \gg a$, we get in the harmonic limit

$$
e(t)=\frac{1}{16 \pi}\left(\frac{2 T_{2}}{a^{2}}+\frac{T_{i}-T_{1}}{\ell_{T_{2}}^{2}\left(t-t_{1}\right)+\ell_{T_{1}}^{2}\left(t_{1}\right)}+\frac{T_{1}-T_{2}}{\ell_{T_{2}}^{2}\left(t-t_{1}\right)}\right) .
$$




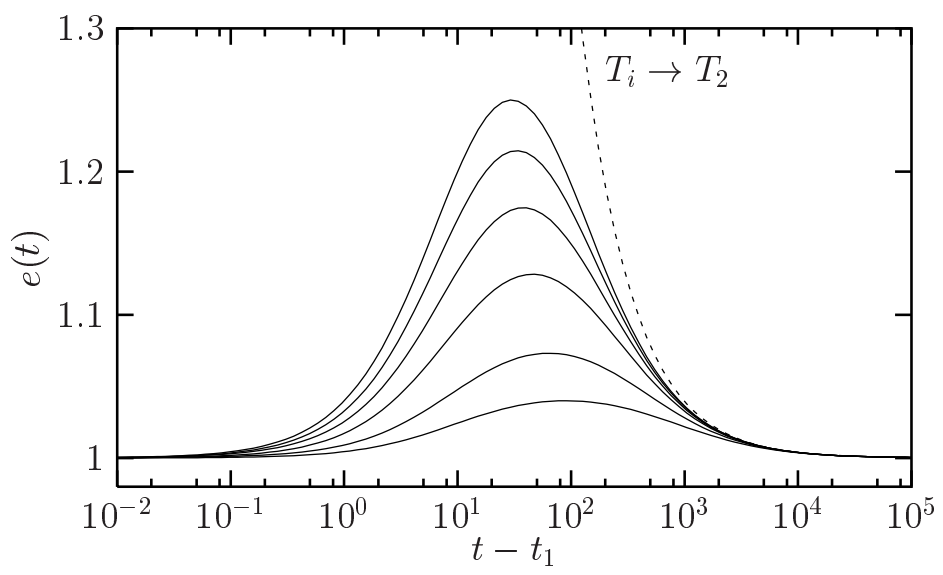

Fig. 2 - Kovacs effect in the 2D XY model. Full lines are for various intermediate temperature $T_{1}=0.95,0.9,0.8,0.7,0.6,0.5$ (from bottom to top), with $T_{i}=5.0$ and $T_{2}=1.0$.

The energy first increases due to the third term, since $\eta_{1}<\eta_{2}$. When $\ell_{T_{2}}\left(t-t_{1}\right) \gg \ell_{T_{1}}\left(t_{1}\right)$, the second and third terms combine to give the simple ageing behaviour of a system directly quenched from $T_{i}$ to $T_{2}$, and hence a decreasing energy density which merges with the curve obtained in this simple experiment. Equation (6) was used to build the curves in Fig. 2, which reproduce quantitatively experimental findings.

Immediately after the shift at time $t_{1}$, fluctuations with $k^{-1} \ll \ell_{T_{1}}\left(t_{1}\right)$ are equilibrated at $T_{1}$ and have to increase their energy in order to equilibrate at the new temperature $T_{2}$. Fluctuations with $\ell_{T_{1}}\left(t_{1}\right) \ll k^{-1}$, which retained their nonequilibrium state at $T_{i}$, decrease their energy to reach their equilibrium state at $T_{2}$. These two types of fluctuations have opposite contributions to the energy evolution and act on different time scales. Thus, a maximum occurs at a time $t_{\mathrm{k}}$, such that $\ell_{T_{2}}\left(t_{\mathrm{k}}-t_{1}\right) \sim \ell_{T_{1}}\left(t_{1}\right)$, the larger $T_{2}-T_{1}$ the larger the height of the maximum. This interpretation can be viewed as a spatial transposition of the distribution of time scales usually invoked to account for this effect 11 .

Discussion. - We have shown that coarsening models at criticality reproduce generic ageing, rejuvenation, memory and Kovacs effects seen in many glassy systems, thus providing a new and simple phenomenological domain growth approach to ageing phenomena.

This seems to beg the question: are glassy systems critical? A possible answer is yes they are, in which case glassy systems should have in common a line of critical points below the glass transition, in analogy with the 2D XY model. This is not inconsistent with existing theoretical descriptions of the spin glass phase [10], but seems less likely in other systems, such as disordered ferromagnets [7] or ferroelectrics [5]. Another is no; but they do at least behave as if there is a line of critical points over the experimental time window. The second, more pragmatic solution requires that the coherence length $\ell_{T}(t)$ is never decoupled from $\xi_{\text {eq }}(T)$, even when the latter is finite. One might argue this if the excitations on length scales exceeding $\xi_{\text {eq }}(T)$ are dominated by activated processes, giving exponentially increasing time scales for $\ell_{T}(t) \geq \xi_{\text {eq }}$. These conditions would make it virtually impossible to enter a regime where $\ell_{T}(t) \gg \xi_{\text {eq }}(T)$. In this case, a slow evolution in the crossover region could result in effective critical behaviour with continuous evolution of the exponents, with the temperature, which is the main ingredient of our analysis of the rejuvenation effect. This is fully consistent with results in 3D spin glasses [29, 30]. It also implies that similar results could be obtained 
in spin glass films around the $T=0$ critical point 31.

In both cases, of course, disorder is essential. Indeed, we would not expect to observe ageing phenomena easily in a non-disordered critical system as the divergence of time scales is actually very weak and can in general be beaten easily, both experimentally and numerically. Disorder would therefore be needed to provide the necessary separation of time scales [15] and to put the ageing phenomena discussed here within the observed experimental time window.

\section{REFERENCES}

[1] Struik L. C. E., Physical aging in amorphous polymers and other materials (Elsevier, Amsterdam) 1978.

[2] Nordblad P. And Svendlidh P., in Spin Glasses and Random Fields, edited by A. P. Young, Vol. 12 (World Scientific, Singapore) 1998, p. 1.

[3] Papadopoulou E. L. and Nordblad P., Eur. Phys. J. B, 22 (2001) 187.

[4] Cipelletti L. et Al., Phys. Rev. Lett., 84 (2000) 2275; Cloitre M. et Al., Phys. Rev. Lett., 85 (2000) 4819; Knaebel A. et Al., Europhys. Lett., 52 (2000) 73; DÉrec C. et Al., C. $R$. Acad. Sci. Paris IV, 1 (2000) 1115; Ramos L. ET AL., Phys. Rev. Lett., 87 (2001) 245503.

[5] Alberici-Kious F. ET Al., Phys. Rev. Lett., 81 (1998) 4987.

[6] Colla E. V. et Al., Phys. Rev. B, 63 (2001) 134107.

[7] Vincent E., Dupuis V., Alba M., Hammann J. and Bouchaud J.-P., Europhys. Lett., 50 (2000) 674.

[8] Vaknin A., Ovadyahu Z., and Pollak M., Phys. Rev. Lett., 84 (2000) 3402.

[9] Josserand C. ET Al., Phys. Rev. Lett., 85 (2000) 3632.

[10] Bouchaud J. P., Cugliandolo L. F., Kurchan J., and Mézard M., in Spin Glasses and Random Fields, edited by A. P. Young, Vol. 12 (World Scientific, Singapore) 1998, p. 161.

[11] Bray A. J., Adv. Phys., 43 (1994) 357.

[12] Cugliandolo L. F. And Dean D. S., J. Phys. A, 28 (1995) 4213.

[13] Yoshino H., Lemaître A., and Bouchaud J. P., Eur. Phys. J. B, 20 (2001) 367.

[14] Bray A. J. And Moore M., Phys. Rev. Lett., 58 (1987) 57.

[15] Bouchaud J. P., in Soft and fragile matter, edited by Cates M. E. And Evans M. R. (Institute of Physics Publishing, Bristol) 2000, p. 285.

[16] Hammann J. et Al., Physica A, 185 (1992) 278.

[17] Bouchaud J. P. And Dean D. S., J. Phys. I (France), 5 (1995) 265; Sasaki M. And Nemoto K., J. Phys. Soc. Jpn, 69 (2000) 2283.

[18] Cugliandolo L. F. and Kurchan J., J. Phys. A, 27 (1994) 5749; Phys. Rev. B, 60 (1999) 922.

[19] Berezinskit V. L., Sov. Phy. JetP, 32 (1971) 493.

[20] Cugliandolo L. F., Kurchan J., and Parisi G., J. Physique I, 4 (1994) 1641.

[21] Berthier L., Holdsworth P. C. W., and Sellitto M., J. Phys. A, 34 (2001) 1805.

[22] Rutenberg A. D. And Bray A. J., Phys. Rev. E, 51 (1995) R1641.

[23] Godrèche C. And Luck J. M., J. Phys. A, 33 (2000) 9141.

[24] Jonason K., Vincent E., Hammann J., Bouchaud J. P., and Nordblad P., Phys. Rev. Lett., 81 (1998) 3243

[25] Bellon L., Ciliberto S., And Laroche C., Europhys. Lett., 51 (2000) 551.

[26] Kovacs A. J., Adv. Polym. Sci., 3 (1963) 394; Kovacs A. J. ET AL., Journal of Polymer Science, 17 (1979) 1097.

[27] Höhler R., Cohen-Addad S., and Asnacios A., Europhys. Lett., 48 (1999) 93.

[28] Leheny R. L. And Nagel S. R., Phys. Rev. B, 57 (1998) 5154.

[29] Berthier L. and Bouchaud J. P., in preparation.

[30] Bouchaud J. P., Dupuis V., Hammann J., and Vincent E., Phys. Rev. B, 65 (2001) 024439.

[31] Mattson J. et Al., Phys. Rev. B, 47 (1993) 14626. 